\title{
A Proof of the Addition Theorem for the Legendre Functions.
}

By Dr T, M. MacRobert.

(Read and Received 7th December 19\%3).

§1. A Theorem of Dougall's. In Vol, XVIII. (p. 78) of these Proceedings Dr Dougall has established the theorem that, if $m$ is a positive integer,

$$
\begin{aligned}
& \frac{\Pi(n-m)}{\Pi(n+m)} P_{n}^{m}(x) P_{n}^{m}\left(x^{\prime}\right) \frac{\pi}{\sin n \pi} \\
& \quad=\sum_{p=m}^{\infty}(-1)^{p}\left\{\frac{1}{n-p}-\frac{1}{n+p+1}\right\} \frac{\Pi(p-m)}{\Pi(p+m)} P_{p}^{m}(x) P_{p}^{m}\left(x^{\prime}\right), \ldots
\end{aligned}
$$

where $x=\cos \theta, x^{\prime}=\cos \theta^{\prime}$. This can be proved by taking the integral

$$
\frac{1}{2 \pi i} \int \frac{\Pi(\zeta-m)}{\Pi(\zeta+m)} \frac{P_{\zeta}^{n}(x) P_{\zeta}^{m}\left(x^{\prime}\right)}{\zeta-n} \frac{\pi}{\sin \zeta \pi} d \zeta
$$

round a circle $|\zeta|=p+\frac{1}{2}$ and making $p \rightarrow \infty$. From the formula

$$
P_{\zeta}^{m}(z)=\frac{\left(z^{2}-1\right)^{\frac{1}{m}}}{2^{m} \Pi(m)} \frac{\Pi(\zeta+m)}{\Pi(\zeta-m)} F\left(m-\zeta, m+\zeta+1, m+1, \frac{1-z}{2}\right)
$$

it is clear that the integrand is holomorphic except at the zeros of $(\zeta-n) \sin (\xi \pi)$, and from the expression (10) of the previous paper in which the series are convergent or asymptotic in $n$ for $0<\theta<\pi$, it can be deduced that the integral round the circle $\rightarrow 0$ as $p \rightarrow \infty$, provided that $0<\theta<\pi, 0<\theta^{\prime}<\pi$, and $\theta+\theta^{\prime}<\pi$.

Similarly from the integral

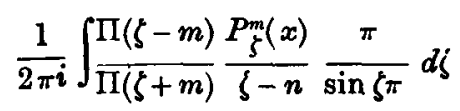

it can be deduced that, for $-1<x<1$,

$$
\begin{aligned}
& \frac{\Pi(n-m)}{\Pi(n+m)} P_{n}^{m}(x) \frac{\pi}{\sin n \pi} \\
& \quad=\sum_{p=m}^{\infty}(-1)^{p}\left\{\frac{1}{n-p}-\frac{1}{n+p+1}\right\} \frac{\Pi(p-m)}{\Pi(p+m)} P_{p}^{m}(x),
\end{aligned}
$$

and, in particular, that, when $m=0$,

$$
P_{n}(x)=\frac{\sin n \pi}{\pi} \sum_{p=0}^{\infty}(-1)^{p}\left\{\frac{1}{n-p}-\frac{1}{n+p+1}\right\} P_{p}(x) .
$$


These formulae may be employed to deduce various formulae for the Legendre Functions from the corresponding formulae for the Legendre Polynomials.

§2. The Addition Theorem. For example, the Addition Theorem for $P_{n}(z)$ when $n$ is not an integer may be obtained in this way from the Addition Formula for the Legendre Polynomials.

Let $z=x x^{\prime}-\sqrt{ }\left(x^{2}-1\right) \sqrt{ }\left(x^{\prime 2}-1\right) \cos \phi$, where $-1<z<1$; then, from (3)

$$
P_{n}(z)=\frac{\sin n \pi}{\pi} \sum_{p=0}^{\infty}(-1)^{p}\left\{\frac{1}{n-p}-\frac{1}{n+p+1}\right\} P_{p}(z) .
$$

Now substitute for $P_{p}(z)$ its expansion (the Addition Formula)

$$
P_{p}(z)=P_{p}(x) P_{p}\left(x^{\prime}\right)+2 \sum_{m=1}^{p}(-1)^{m} \frac{(p-m) !}{(p+m) !} \cos m \phi P_{p}^{m}(x) P_{p}^{m}\left(x^{\prime}\right),
$$

and rearrange the resulting double series; thus

$$
P_{n}(z)=\frac{\sin n \pi}{\pi} \sum_{p=0}^{\infty}(-1)^{p}\left\{\frac{1}{n-p}-\frac{1}{n+p+1}\right\} P_{p}(x) P_{p}\left(x^{\prime}\right)
$$

$$
\begin{aligned}
& +\frac{2 \sin n \pi}{\pi} \sum_{m=1}^{\infty}(-1)^{n} \cos m \phi \sum_{p=m}^{\infty}(-1)^{\frac{1}{x}} \frac{(p-m) !}{(p+m) !}\left\{\frac{1}{n-p}-\frac{1}{n+p+1}\right\} P_{p}^{m}(x) P_{p}^{m}\left(x^{\prime}\right) \\
& =P_{n}(x) P_{n}\left(x^{\prime}\right)+2 \sum_{m=1}^{\infty}(-1)^{m} \cos m \phi \frac{\Pi(n-m)}{\Pi(n+m)} P_{n}^{m}(x) P_{n}^{m}\left(x^{\prime}\right) \ldots \ldots \ldots \ldots \ldots(4)
\end{aligned}
$$

by (1). This is the Addition Theorem; it is valid for all values of $x$ and $x^{\prime}$ for which the series (4) is convergent. 